\title{
Fibrous Meningioma with Skull Invasion
}

\author{
Shinya Ichimura ${ }^{1}$ Kento Takahara ${ }^{1}$ Koji Fujii ${ }^{1}$ \\ ${ }^{1}$ Department of Neurosurgery, Shizuoka City Shimizu Hospital, \\ Shizuoka, Japan
}

\begin{abstract}
Address for correspondence Shinya Ichimura, MD, Department of Neurosurgery, Shizuoka City Shimizu Hospital, 1231 Miyakami Shimizu-Ku Shizuoka-City, Shizuoka 424-8636, Japan (e-mail: shinya3917@gmail.com).
\end{abstract}

\begin{abstract}
Keywords

- fibrous meningioma

- skull invasion

- osteopontin

- matrix metalloproteinase-2

- integrin $\beta-1$

In patients with meningiomas, the presence of skull invasion is known to be a predictor of aggressive clinical behavior, which may negatively influence patient outcomes. In the present report, we discuss a case of fibrous meningioma with skull invasion. A 42-year-old woman was referred to our department presenting with hyperostosis in the right parietal bone. T1-weighted magnetic resonance imaging with gadolinium enhancement revealed prominent enhancement of the intraosseous lesion and dura mater. Following the removal of the tumor body and bone lesion, we performed immunohistochemical staining for osteopontin (OPN), matrix metalloproteinase-2 (MMP2), and integrin $\beta-1$ (CD29). The tumor body was immunoreactive for OPN and CD29, but not MMP2, whereas, the bone lesion was immunoreactive for all the three antigens. The present case suggests that OPN, MMP2, and CD29 play key regulatory roles in bone invasion.
\end{abstract}

\section{Introduction}

The majority of meningiomas are benign tumors, $\sim 5 \%$ of the tumors exhibiting the features of malignancy. ${ }^{1,2}$ Although the meningiomas invading the bone are considered pathologically benign, they may infiltrate adjacent neural and soft tissues, becoming much larger than the original dural tumor. ${ }^{2}$

Hyperostosis is a well-known symptom of bone-invading meningiomas, suggesting preferential bone tropism by meningioma cells. Osteopontin (OPN), matrix metalloproteinase-2 (MMP2), and integrin $\beta-1$ (CD29) are the key regulatory mediators in the pathogenesis of bone invasion and osteolytic metastasis. ${ }^{2}$ Here, we discuss a case of fibrous meningioma with skull invasion with unequivocal immunoreactive expression of OPN, MMP2, and CD29 observed in the tumor cells.

\section{Case Presentation}

A 42-year-old woman presenting with dizziness was diagnosed with hyperostosis of the right parietal bone by computed tomography (-Fig. 1A). She was referred in good general health and without any history of trauma. T1-weighted magnetic resonance imaging with gadolinium enhancement revealed prominent enhancement of the intraosseous lesion and dura mater ( - Fig. 1B).

The patient underwent surgery in the supine lateral position, under general anesthesia. A periosteum flap was made after retracting the scalp. Hyperostosis of the skull was observed ( - Fig. 2A), and a right parietal craniotomy was performed with a $2 \mathrm{~cm}$ margin around the lesion, revealing a tumor in the dura mater $(-$ Fig. 2B). The tumor was completely excised with $\mathrm{a} \geq 1 \mathrm{~cm}$ margin. After removing the dura surrounding the lesion, the dural defect was patched with the periosteum flap, and the scalp was sutured. The postoperative course was uneventful. Twelve days after the tumor was removed, a cranioplasty, for bone defect, was performed using a customized, artificial, porous hydroxyapatite bone flap (APACERAM, Hoya Technosurgical Corporation, Tokyo, Japan) (-Fig. 2C).

\section{Pathological Findings}

Histologically, the tumor attached to the dura mater constituted small sheets and nests of polygonal- to spindle-shaped arachnoid cells, abundant dense fibrohyalinous stroma, and scattered psammoma bodies. No cell atypia or mitotic figures were observed ( - Fig. 3A). The tumor had invaded the skull, and consequently, thick osteoplastic bone trabeculae and 
thick fibrous tumor tissue had obliterated the marrow space (-Fig. 3B). The MIB-1 staining index of the meningioma was $<2.0 \%$, suggesting low proliferative potential. Thus, the patient was diagnosed with fibrous meningioma with skull invasion and focal bone marrow hypercellularity.

We also examined the expression profiles of OPN (1:200, Anti-Human Osteopontine, OP3N), MMP2 (1:200, Anti-Human MMP2, 17B11), and CD29 (dilution 1:100, Anti-Human CD29, 7F10). All antibodies were monoclonal, raised in mouse

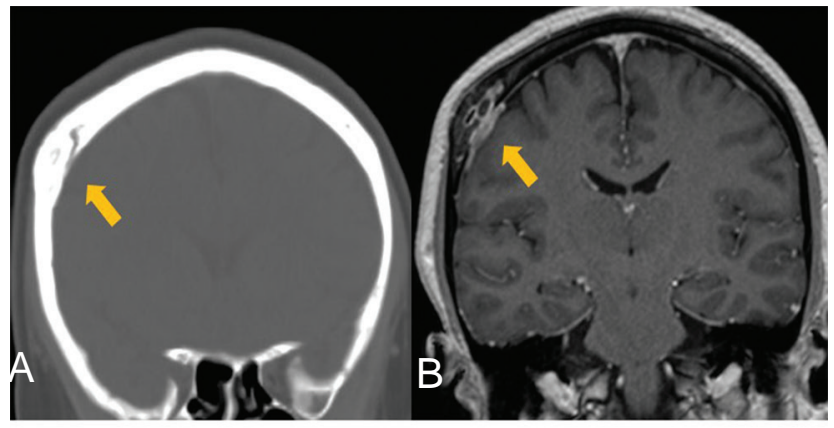

Fig. 1 (A) Computed tomography showing hyperostosis in the right parietal bone on coronal image. (B) T1-weighted magnetic resonance images with gadolinium enhancement showing enhancement of the intraosseous lesion and dura mater. Arrows indicate lesions. hybridoma clones (Leica Biosystems Newcastle Ltd, Newcastle, UK) (-Fig. 4). Tumor cells in both the dural matter and skull were strongly immunoreactive for OPN ( - Fig. 4A) and CD29 ( - Fig. 4C), but not for MMP2 ( - Fig. 4B). The endothelial cells were only immunoreactive for CD29. However, fibroblast-like spindle cells in the bone lesion were unequivocally immunoreactive for OPN, MMP2, and CD29 ( - Fig. 4E and F).

\section{Discussion}

Meningiomas are the predominant intracranial tumors, accounting for $\sim 25 \%$ of all intracranial tumors. Rarely, extradural "ectopic" tumors have also been reported, ${ }^{1}$ accounting $\sim 1$ to $2 \%$ of all meningiomas. ${ }^{1}$ Owing to their rarity, the ectopic meningiomas of the skull are not primarily suspected on preoperative diagnosis. Instead, more typical differential diagnoses include fibrous dysplasia and osteoid osteoma, which represent the most common benign primary tumors. ${ }^{1}$

The presence of brain invasion is a predictor of aggressive clinical behavior and recurrence, and tumors exhibiting brain invasion are designated as grade II, in accordance with World Health Organization (WHO) classification criteria. ${ }^{3}$ Although the WHO criteria do not consider cases with skull invasion to be atypical, the extent of skull invasion can directly influence the clinical behavior of meningiomas, as well as the patient

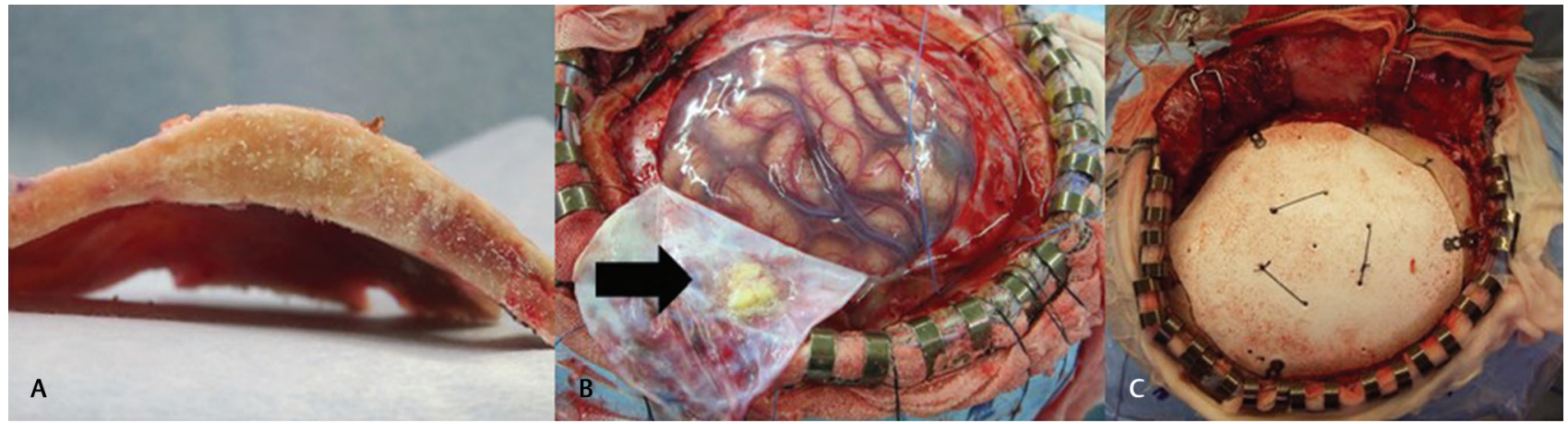

Fig. 2 Intraoperative findings. (A) Hyperostosis was observed in the bone flap. (B) The tumor was observed in the dura mater. (C) Cranioplasty was performed 12 days after tumor removal using an artificial hydroxyapatite bone flap (APACERAM, Hoya Technosurgical Corporation, Tokyo, Japan). Arrow indicates tumor.

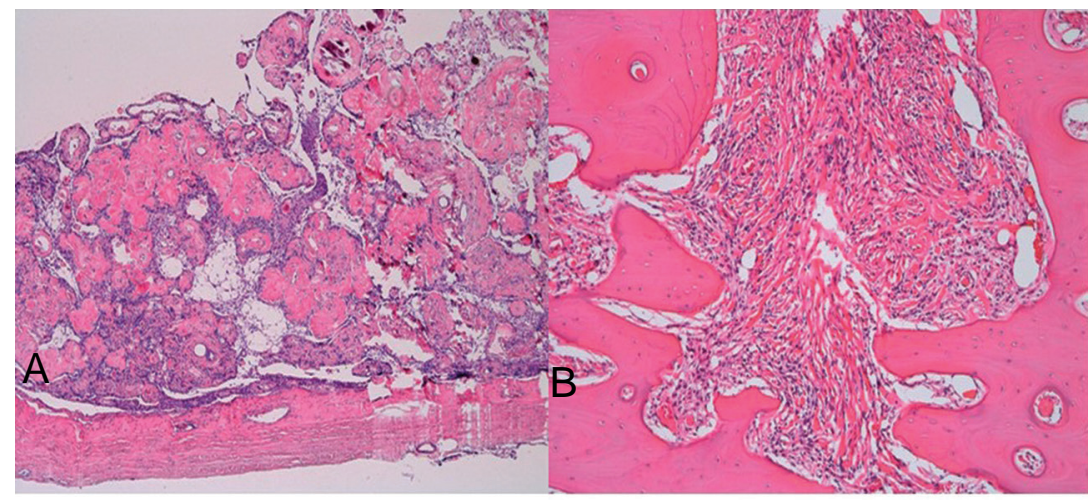

Fig. 3 (A) The tumor at the dura mater was composed of spindle-shaped cells and abundant fibrohyalinous stroma with scattered psammoma bodies. (B) Hematoxylin and eosin staining revealed that the bone marrow space at the invasion site was obliterated, with thick bone trabeculae and tumor tissue. 


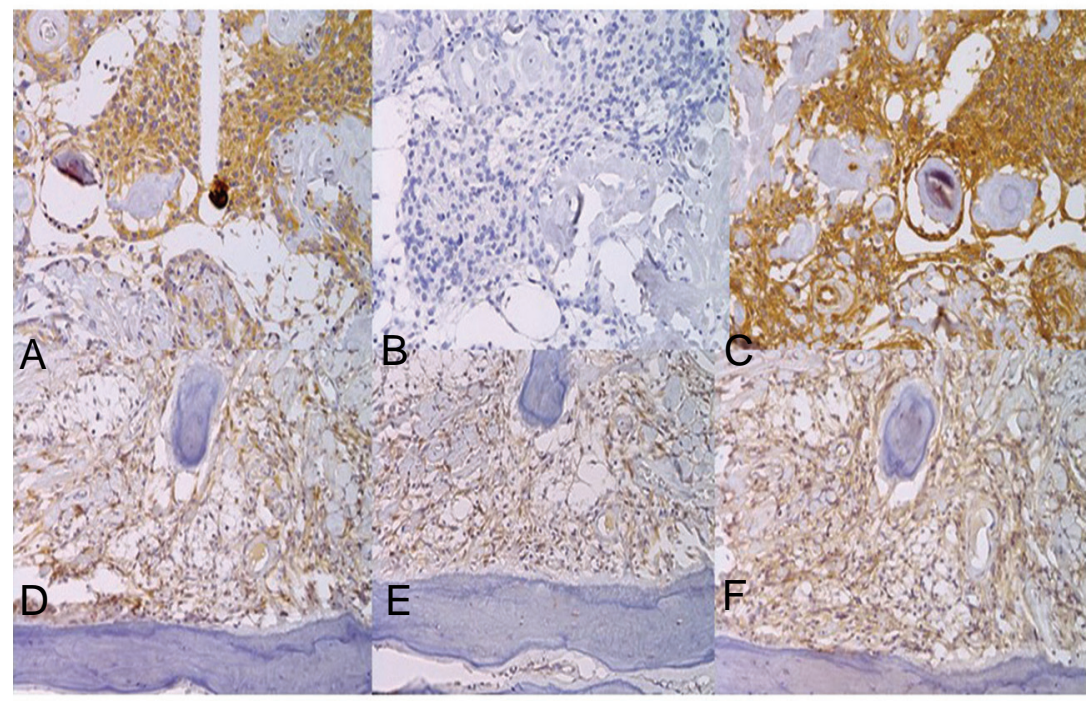

Fig. 4 The tumor cells were immunopositive for osteopontin (OPN) (A) and matrix metalloproteinase-2 (MMP2) (C), but not for integrin $\beta$-1 (CD29) (B) at the dura mater, whereas strong immunopositivity for all three was observed in the skull lesion (D; OPN, E; MMP2, F; CD29).

outcomes. $^{2}$ Bone-invading meningiomas are clinically challenging, as incomplete tumor resection presents an increased risk of both disease recurrence and compromising the vital vascular and neural structures. ${ }^{2}$

Tumor invasion into the adjacent tissue may hamper radical resection and reconstruction by simple surgical means. ${ }^{4}$ In such cases, reconstruction of the skull is problematic due to tissue deficits. Although there are many alternatives (autografts, allografts), we used a custom-made, artificial, porous hydroxyapatite bone flap in our patient. ${ }^{4}$ Hydroxyapatite implants have osteoconductive and biocompatible properties conferring good osseointegration with the cranial vault. ${ }^{5}$ The porous nature of the implant allows ingrowth of the osteoprogenitor cells and increases the resistance of the implant. ${ }^{5}$

The molecular mechanisms underlying tumor growth and malignant progression are poorly understood, with relevant investigations yielding discordant results, partly because meningiomas are a large, heterogeneous group classified based on histological findings, and present uncommon molecular or genetic traits. ${ }^{2}$ However, the molecular regulators of bone tropism, osteolytic activity, and vascular remodeling are dependent on the anatomical location of the meningioma. ${ }^{2}$

Presently, the tumor cells in both the dura mater and skull were strongly immunoreactive for OPN and CD29, but not MMP2, whereas, the cells in the bone lesion were immunoreactive for all the three antigens. OPN increases osteoclast-mediated bone resorption in breast carcinomas. ${ }^{6}$ OPN has been implicated in bone invasion in several types of cancers and intracranial tumors. ${ }^{7}$

MMPs are a family of zinc-dependent peptidases that mediate the degradation of the extracellular matrix components, and implicated in tumor cell growth, invasion, and metastasis. ${ }^{2}$ Previous studies focused on establishing the role of MMP2 expression in meningiomas with tumor recurrence. ${ }^{2}$ MMP2 expression was the weakest in meningothelial meningiomas and strongest in fibroblastic meningiomas. ${ }^{8}$
Integrins mediate bone metastasis and osteoclast activity in breast and prostate neoplasms. ${ }^{9,10}$ These proteins mediate the adhesion of osteoclasts to the bone matrix in breast cancer, stimulating the release of lysosomal enzymes that trigger bone collagen degradation. ${ }^{10}$ However, a few studies have focused on CD29 expression in meningiomas. ${ }^{9}$ CD29 expression was found higher in atypical and malignant meningioma vasculature and tumor cells than in benign meningiomas. ${ }^{9}$ CD29 expression has also been observed in the vessels present in the peritumoral brain tissue, suggesting its abnormal activation in the vasculature surrounding the tumor tissue, as normal brain tissue does not express CD29. ${ }^{2}$

\section{Funding}

None.

\section{Conflict of Interest}

None declared.

\section{Acknowledgments}

The authors would like to thank to BML Group PCL JAPAN (Tokyo, Japan), as well as Mr. Seiji Matsumoto at the Department of Pathology, Shizuoka City Shimizu Hospital, for assistance with histological staining in this study.

\section{References}

1 Al-Mefty O, Smith R. Tuberculum sellae meningiomas. In: Al-Mefty O, ed. Meningiomas. New York, NY: Raven, Ltd; 1991 395-411

2 Salehi F, Jalali S, Alkins R, et al. Proteins involved in regulating bone invasion in skull base meningiomas. Acta Neurochir (Wien) 2013;155(3):421-427

3 Sade B, Chahlavi A, Krishnaney A, Nagel S, Choi E, Lee JH. World Health Organization Grades II and III meningiomas are rare in the cranial base and spine. Neurosurgery 2007;61(6):1194-1198

4 Moyer JS, Chepeha DB, Teknos TN. Contemporary skull base reconstruction. Curr Opin Otolaryngol Head Neck Surg 2004;12(4):294-299 
5 Fricia M, Nicolosi F, Ganau M, et al. Cranioplasty with porous hydroxyapatite custom-made bone flap: results from a multi-centre study enrolling 149 patients over 15 years. World Neurosurg 2019;121:160-165

6 Macrì A, Versaci A, Lupo G, et al. Role of osteopontin in breast cancer patients. Tumori 2009;95(1):48-52

7 Tremblay P, Beaudet MJ, Tremblay E, Rueda N, Thomas T, Vallières L. Matrix metalloproteinase 2 attenuates brain tumour growth, while promoting macrophage recruitment and vascular repair. J Pathol 2011;224(2):222-233

8 Rooprai HK, van Meter TE, Robinson SDF, King A, Rucklidge GJ, Pilkington GJ. Expression of MMP-2 and -9 in short-term cultures of meningioma: influence of histological subtype. Int J Mol Med 2003;12(6):977-981

9 Bello L, Zhang J, Nikas DC, et al. Alpha(v)beta3 and alpha(v) beta5 integrin expression in meningiomas. Neurosurgery 2000;47(5):1185-1195

10 van der Horst G, van den Hoogen C, Buijs JT, et al. Targeting of. $\alpha$. (v)-integrins in stem/progenitor cells and supportive microenvironment impairs bone metastasis in human prostate cancer. Neoplasia 2011;13(6):516-525 